\title{
Studies of grain segregation patterns on a destoner using a CFD-DEM approach
}

\author{
A. S. Kannan ${ }^{1}$, M. A. E. Hansen ${ }^{2}$, J. M. Carstensen ${ }^{2}$, P. Thorn ${ }^{3}$, \\ J. Lund ${ }^{3} \&$ S. Sasic ${ }^{1}$ \\ ${ }^{I}$ Department of Applied Mechanics, Chalmers University of Technology, \\ Sweden \\ ${ }^{2}$ Videometer A/S, Denmark \\ ${ }^{3}$ Westrup A/S, Denmark
}

\begin{abstract}
Removal of contaminants from 'food grade' quality grains is of great importance in food and grain processing operations. A thorough understanding of the inherent granular segregation profiles on this processing equipment is a pivotal step in the design and development of more efficient processes. One such grain cleaning operation is the 'density-based separation' using a destoner. This process removes stones and other heavy material from lighter food grains using a vibrating deck and fluidizing air. In this paper we formulate a CFD-DEM framework (set up and implemented in the OpenFOAM ${ }^{\circledR}$ environment) to study granular segregation patterns on a destoner. The scheme is first validated by comparing simulations with experimental data using a gas-solid fluidized-bed test case. A good agreement between the experiments and the simulations is noted. This proposed framework is then used to characterize the combined effects of deck inclination and fluidization velocities on the separation profiles generated from a virtual destoner. We have found these profiles to be highly sensitive to changes in fluidization conditions, with the gradual development of segregation zones at velocities close to the minimum fluidization velocity of the heavier component. A deck inclination of 5 degrees and a fluidization velocity of $2.0 \mathrm{~m} / \mathrm{s}$ is considered optimal while steeper slopes (inclinations of 15 degrees) and lower air velocities ( 0 and $1.5 \mathrm{~m} / \mathrm{s}$ ) are deemed unsuitable for segregation.

Keywords: destoner, grain cleaning operation, OpenFOAM ${ }^{\circledR}, C F D-D E M$, deck inclination, fluidization.
\end{abstract}




\section{Introduction}

In today's fast-paced world, the increasing demands on the requirements of the human population have led to the development of numerous applications for the rapid and large-scale processing of food crops. Ensuring the highest quality of the grains meant for large-scale cultivation is hence an incumbent criterion that should be fulfilled. Historically, 'winnowing' has been employed to remove bran and stones from the harvested crop and, over the years, this ancient practice has been refined and developed to give rise to modern machinery, which effortlessly clean harvested crops. A wide variety of machinery operating under the fundamental principle of separation based on 'specific gravity or density' are available today and are widely employed in the downstream processing of food grains. The 'destoner' is an example of one such equipment that is utilized in the preliminary stages of grain downstream processing to remove stone and other heavier contaminants from food grade grains. It is of common interest to obtain a fundamental understanding of the underlying phenomena of granular separation in order to develop smarter technologies for the cleaning of food grains. The purpose of this work is thus to look at such phenomena using numerical methods.

Destoners employ air as the separating standard, widening the relative difference between particles of differing weights (grains rise or fall by their relative weight to air). An oscillating deck transports the heavier contaminant (e.g. stones) towards the higher end, and the lighter grain is collected at the base. The segregation patterns on the deck of a destoner are principally controlled by two main variables: fluidizing air and deck tilt. The dependence of the performance of the machine on just these two parameters enables tighter control on quality and relative ease of operation. The lack of prior work on destoners can be supplemented by collating results from other closely related applications such as shaking separation of rice and paddy.

The building blocks of a comprehensive model to describe granular segregation in a destoner can be obtained by examining these related studies on shaking separation of rice. The movement of paddy and rice in opposite directions on an oscillating tray separator was first described by Das [1] as a result of different coefficients of friction between paddy and the deck, between rice and the deck and between paddy and rice. A similar mechanism can be adopted for destoners, with separating particles having similarity in shape but differing in specific gravity and surface characteristics. These studies highlight the fact that gravity separators (such as the destoners) are driven by contactdominated (dense) granular flows and a suitable description of the system can be obtained by applying the corresponding mechanical models.

In the current age of computers, there is a noticeable shift towards in-silico description of systems in order to obtain an insight into previously unknown phenomena by solving the fundamental equations of conservation of mass, momentum, energy etc. One such effort - the Discrete Elementary Method (or DEM), developed by Cundall and Strack [2], is a well-established method (based on contact mechanical treatment) that can provide dynamic information, such as 
trajectories of individual particles and transient forces acting on them. Similar information is exceptionally difficult, if not impossible, to obtain by physical experimentation (Zhu et al. [3]). Consequently, this method has found increasing application in the field of grain downstream processing.

The application of DEM to agricultural and food particulates is limited to rather simplified case studies (dealing with the behaviour of a few hundred particles) including numerical studies on the separation process of soybeans and mustard seeds by a vibrating screen (Li et al. [4]) and simulation of the shaking separation of paddy and brown rice (Sakaguchi et.al. [5]). So far, the latter work represents the only validated application of DEM in grain downstream. This model was validated against experimental results at the macroscopic scale demonstrating the capabilities of DEM as a tool to study granular flow phenomena of food particulates (Sakaguchi et al. [5]).

\section{Simulation method}

Prior efforts in DEM of grain downstream processing were limited to describing the gravity flow of a solid particulate bed without examining the influence of the surrounding continuum (air). A more inclusive particle-fluid model would provide new insights into the bulk behaviour of food particulates during seed cleaning applications. The current work aims to formulate such a CFD-DEM scheme to study granular segregation patterns on a destoner.

\subsection{CFD-DEM framework}

The DEM is a powerful tool that provides a valuable insight into particle 'microscale' behaviour enabling a better understanding of the bulk system behaviour (macro-scale). Coupled with CFD, this framework is capable of resolving particle-fluid 'micro' phenomena aiding in mapping several pertinent transport fields (such as thermal gradients, concentration profiles, velocity fields etc.) crucial to a wide range of industrial applications. The CFD-DEM approach proposed by Tsuji et al. [6] is one of the most widely used research tools to study granular flows. In this approach the motion of individual particles is obtained by solving Newton's second law of motion (for both translation and rotation), whereas the continuum flow is resolved by solving the locally averaged NavierStokes equations. Thus, the equation governing the translational motion of particle $i$ is given as

$$
m_{i} \frac{d v_{i}}{d t}=\boldsymbol{f}_{f, i}+\sum_{j=1}^{k_{i}}\left(\boldsymbol{f}_{c, i j}+\boldsymbol{f}_{d, i j}\right)+m_{i} \boldsymbol{g},
$$

where $m_{i}$ and $v_{i}$ are, respectively, the mass and velocity of particle $i$, and $k_{i}$ is the number of particles in contact with the tracked particle. The forces involved are: the fluid-particle interaction force (the drag force), $\boldsymbol{f}_{f, i}$, the gravitational force, $m_{i} \boldsymbol{g}$, and the inter-particle forces which include the contact force, $\boldsymbol{f}_{c, i j}$, and viscous contact damping force $\boldsymbol{f}_{d, i j}$. The drag force is determined at the individual particle level and is hence not only dependent on the relative velocity $(|\boldsymbol{u}-\boldsymbol{v}|)$ between the fluid and particle but also on the presence of the surrounding particles. The empirical Ergun-Wen Yu drag correlation (eqn (2)) is used in the current work 
with the effect of the presence of other particles considered in terms of local porosity $\left(\varepsilon_{f}\right)($ Zhu et al. [3]).

$$
\begin{gathered}
\boldsymbol{f}_{f, i}=\beta_{p f}(\boldsymbol{u}-\boldsymbol{v}) / \rho_{f} \\
\beta_{p f}=150 \frac{\left(1-\varepsilon_{f}\right)^{2}}{\varepsilon_{f}} \frac{\mu_{f}}{\left(\phi_{p} d_{p}\right)^{2}}+1.75\left(1-\varepsilon_{f}\right) \frac{\rho_{f}}{\left(\phi_{p} d_{p}\right)}|\boldsymbol{u}-\boldsymbol{v}| \quad\left(\epsilon_{f} \leq 0.8\right) \\
\beta_{p f}=\frac{3}{4} C_{d} \frac{|\boldsymbol{u}-\boldsymbol{v}| \rho_{f}\left(1-\varepsilon_{f}\right)}{d_{p}} \varepsilon_{f}^{-2.7} \quad\left(\epsilon_{f}>0.8\right)
\end{gathered}
$$

The inter-particle forces will generate a torque $\left(\boldsymbol{\tau}_{i j}\right)$ causing the particle $i$ to rotate.

$$
I_{i} \frac{d \omega_{i}}{d t}=\sum_{j=1}^{k_{i}} \boldsymbol{\tau}_{i j}
$$

where $\omega_{i}$ is the angular velocity, and $I_{i}$ is the moment of inertia of the particle $i$, given as

$$
I_{i}=\frac{2}{5} m_{i} R_{i}^{2} .
$$

The resolution of granular motion within DEM is dependent on the treatment of collisions between the particles (the contact force, $\boldsymbol{f}_{c, i j}$ ). There are two widely used approaches to handle particle contact: the hard-sphere and the soft-sphere models. The hard-sphere approach is applicable for relatively dilute gas-solid systems as it accounts only for instantaneous and binary collisions. The softsphere model, on the other hand, takes multiple particle collisions into consideration and is commonly used to simulate dense gas-particle systems (Crowe et al. [7]). Thus, in the present work (as we deal with dense granular flow of grains), the soft-sphere model is employed to calculate the contact forces, including the normal, damping and tangential forces between the particles and between particles and walls. The contact forces are modeled using an analogy with a mechanical system consisting of springs, dashpots and friction sliders. The gas phase is treated as a continuous phase and the governing equations are solved using the Finite Volume Method. The continuous phase equations are:

$$
\begin{gathered}
\frac{\partial \varepsilon_{f}}{\partial \tau}+\nabla \cdot\left(\varepsilon_{f} \boldsymbol{u}_{f}\right)=0, \\
\frac{\partial\left(\rho_{f} \varepsilon_{f} \boldsymbol{u}_{f}\right)}{\partial \tau}+\nabla \cdot\left(\rho_{f} \varepsilon_{f} \boldsymbol{u}_{f} \boldsymbol{u}_{f}\right)=-\varepsilon_{f} \nabla p-\boldsymbol{F}_{A}+\nabla \cdot\left(\varepsilon_{f} \boldsymbol{T}\right)+\rho_{f} \varepsilon_{f} \boldsymbol{g},
\end{gathered}
$$

where $\boldsymbol{u}_{f}$ and $p$ are, respectively, the fluid velocity and pressure; $\boldsymbol{T}$ and $\varepsilon_{f}$ are the fluid viscous stress tensor and porosity, respectively. The force $\boldsymbol{F}_{A}$ is the volumetric fluid-particle interaction force (coupling) calculated on the basis of Newton's third law of motion, i.e., the fluid drag force acting on the individual particles from the fluid phase will react on the fluid phase from the individual particles (the fluid-particle drag is summed over a computational cell). In this work, the coupled CFD-DEM framework is implemented into an Open-source computational platform (OpenFOAM). 


\subsection{The virtual destoner (simulation conditions)}

The proposed CFD-DEM framework is applied here to describe the segregation patterns on a destoner. Commercial destoners have a rather simple design consisting of an inclined vibrating deck that is fed with contaminated granular material. Air is forced through tiny openings on the deck (figure 1) fluidizing the granular material above. The heavier contaminants (such as stones) are not fluidized and are carried to the higher side (steeper end) due to the vibrating action of the deck, while the fluidized (lighter) grains move towards the lower end of the deck and are collected there. A schematic of the destoner is provided in figure 1. In order to minimize the computational effort needed to resolve granular flow patterns on a destoner, a symmetric transverse section (as shown in figure 1) is simulated. As indicated above, this work aims at developing a framework to study granular flow behaviour on gravity separators (such as the destoners) and a scaled- down simulation is a logical first step towards this objective.
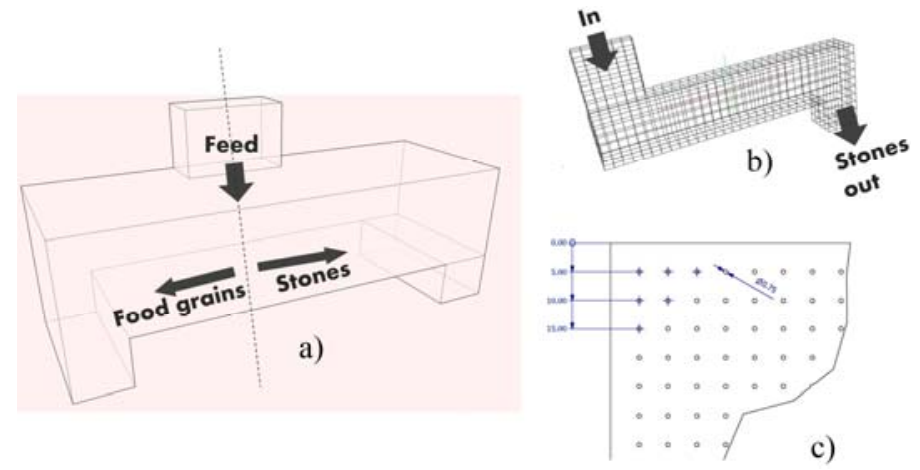

Figure 1: (a) Schematic of a destoner, (b) Transverse symmetric cut of the destoner (geometry that is simulated) and (c) Perforations on the destoner deck.

The simulated domain is dimensioned at $15 \mathrm{~cm} \times 3 \mathrm{~cm} \times 10 \mathrm{~cm}$. The simulated feed consists of spherical particles of different sizes and densities (to mimic stones and food grains) introduced batch-wise. The stones are modelled as denser food grains with all other micro properties similar to that of the grains (Young' modulus, Poisson ration etc.) (table 1). The fluidizing air is introduced into the system by means of a boundary condition that fixes the superficial velocity of air over the deck surface. Additionally, the vibrating action of the deck is approximated to have an amplitude of $1.5 \mathrm{~cm} / \mathrm{s}$ (estimates from manufacturer). The time step used in the numerical simulation is determined from the DEM constraints (as the length scales simulated in DEM are much smaller than those of the resolution of the continuous phase). The simulation conditions, together with the micro-properties of the granular material that are simulated in this study, are provided in table 1. 
Table 1: Simulation conditions.

\begin{tabular}{|c|c|c|c|}
\hline \multirow{2}{*}{$\begin{array}{l}\text { Particle micro-properties } \\
\text { Food grain (modelled on the } \\
\text { basis of rapeseed) }\end{array}$} & & \multicolumn{2}{|c|}{ Continuum properties (air at $20^{\circ} \mathrm{C}$ ) } \\
\hline & & Viscosity $\left(\mathrm{kg} \mathrm{m}^{-1} \mathrm{~s}^{-1}\right)$ & $1.8 \times 10^{-5}$ \\
\hline Density $\left(\mathrm{kg} \mathrm{m}^{-3}\right)^{*}$ & 1100 & Density $\left(\mathrm{kg} \mathrm{m}^{-3}\right)$ & 1.205 \\
\hline Young's Modulus $\left(\mathrm{Nm}^{-2}\right)^{*}$ & $\begin{array}{l}5.0 \mathrm{x} \\
10^{6}\end{array}$ & Fluidizing air velocity $\left(\mathrm{m} \mathrm{s}^{-1}\right)$ & 1.5 \\
\hline Poisson ratio $\left(\mathrm{Nm}^{-2}\right)^{*}$ & 0.25 & Time step (s) & $2.5 \times 10^{-5}$ \\
\hline Coefficient of restitution ${ }^{*}$ & 0.6 & & \\
\hline Coefficient of sliding friction ${ }^{*}$ & 0.3 & & \\
\hline Particle diameter $(\mathrm{mm})$ & 1 & & \\
\hline Number of particles & 4,000 & & \\
\hline \multicolumn{4}{|l|}{$\begin{array}{l}\text { Stone (modelled on the basis of } \\
\text { rapeseed) }\end{array}$} \\
\hline Density $\left(\mathrm{kg} \mathrm{m}^{-3}\right)$ & 2,500 & & \\
\hline $\begin{array}{l}\text { Particle diameter }(\mathrm{mm}) \\
\text { (all other micro-properties are } \\
\text { same) }\end{array}$ & 2.5 & & \\
\hline Number of particles & 400 & & \\
\hline
\end{tabular}

\subsection{Principle of operation}

The main principle of operation of a destoner is that it removes stones from a given input feed using a combined effect of deck vibration and fluidization. The inclination of the deck plays a major role in creating the necessary slope needed to convert vertical stratification (achieved by the fluidizing air) into horizontal separation zones along the deck surface. Additionally, the interaction between the denser material (which is not fluidized) and the deck surface is critical in determining the efficiency of separation. Hence, the right vibrational behaviour needs to be accounted for in the numerical simulations. In this study, it is assumed that the grains that are in contact with the table are 'thrown' towards the higher end of the deck. This motion can be visualized using the schematic below.

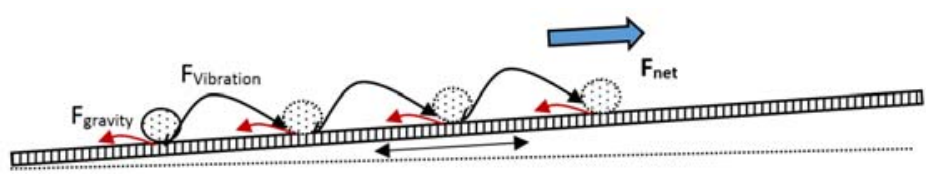

Figure 2: Net grain/stone movement on the deck.

The deck of a real destoner is supplemented with expanded metal surfacing (in order to increase deck friction) to prevent contacting stones from rolling down towards the lower ends of the deck (i.e. for stones $F_{\text {vibration }}>F_{\text {gravity }}$ leading to a net upward propagation). It is assumed that the motion of the deck has negligible effects on the continuum phase, i.e. it only impacts the particulate phase. Hence, a moving or dynamic mesh simulation has not been used in this 
study. Instead, this throwing action of the deck is modelled in a 'static mesh framework' by utilizing a non-zero wall boundary condition on the deck surface (with a constant velocity obtained from measurements on a real destoner). This approach reduces the complexity of the corresponding computations.

\section{Results and discussion}

\subsection{Validation of the DEM framework}

We have first validated our simulation framework using experimental data from literature. For that purpose, we have used the measurements by Goldschmidt et al., performed in a pseudo two-dimensional, laboratory-scale fluidized bed with a simple rectangular geometry and well-defined gas inflow conditions. In this study the experiments were performed with approximately 25,000 glass beads at three different fluidization velocities (Goldschmidt et al. [9]). Note that the lack of experimental data for destoners has necessitated our use of the fluidized-bed test case for validating the DEM framework. In our simulations, we have utilized a domain which is dimensioned at $15 \mathrm{~cm} \mathrm{x} 1.5 \mathrm{~cm} \times 70 \mathrm{~cm}$ and have studied three fluidization velocities $\left(U_{\text {air }}\right)$ of $1.56,1.88$ and $2.50 \mathrm{~m} / \mathrm{s}$, respectively (figure 3 ). These cases match the conditions of the experimental studies by Goldschmidt et al. [9].

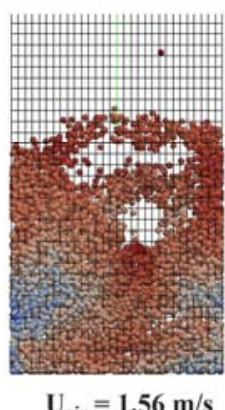

$U_{\text {air }}=1.56 \mathrm{~m} / \mathrm{s}$

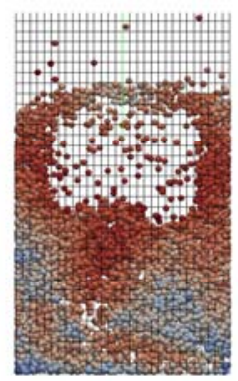

$\mathrm{U}_{\text {air }}=1.88 \mathrm{~m} / \mathrm{s}$

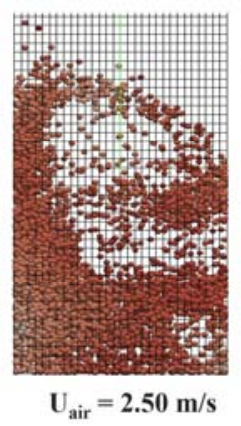

Figure 3: Fluidized bed test cases $(t=5 \mathrm{sec})$ at $\mathrm{U}_{\text {air }}$ of $1.56,1.88$ and $2.50 \mathrm{~m} / \mathrm{s}$ (particles coloured by magnitude of velocity).

The cases were simulated for a total of 5 seconds, after which the corresponding average bed heights $\left(H_{\text {avg }}\right)$ were obtained for each case. This average bed height is then compared with the corresponding data from the experiments by Goldschmidt et al. [9]. This height is calculated based on a percentile estimate of the particle $z$-coordinates (representative of height). The percentile estimate used in the current work indicates the maximum height below which $80 \%$ of the total particle bed reside. The mean of the $80^{\text {th }}$ percentile of particle $z$ position over the time period from 2 (system stabilizes) to 5 seconds (at intervals of 0.25 seconds) is considered as the average particle bed height $\left\langle H_{a v g}\right\rangle$. 


$$
\left\langle H_{\text {avg }}\right\rangle=\frac{\sum_{5}^{t=2} H_{a v g}}{n},
$$

where $n$ is the number of time entries between 2 and 5 seconds at intervals of 0.25 seconds (12 entries). This $80^{\text {th }}$ percentile is considered as a reasonable estimate for the average particle bed height. We study the bed expansion dynamics through the variation of $\left\langle H_{a v g}\right\rangle$ over increasing fluidization velocities (figure 4). Both the experiments and the simulations exhibit a similar trend, with a slight increase followed by stabilization in $\left\langle H_{\text {avg }}\right\rangle$ on increasing the fluidization velocity $\left(U_{\text {air }}\right)$.The error bars in figure 4 represent uncertainties in experimental measurements, and the simulations are within these limits with a slight tendency to over predict average particle bed height $\left\langle H_{a v g}\right\rangle$. This overestimation indicates that further refinements are needed in the DEM framework particularly with the drag law being used, simulated time period (might be too short to obtain statistically significant behaviour) and the contact mechanical parameters (spring-slider-dashpot model parameters) employed. Nevertheless, the overall performance of the DEM framework, i.e. the stability and accuracy of the code, is deemed acceptable under these restrictions.
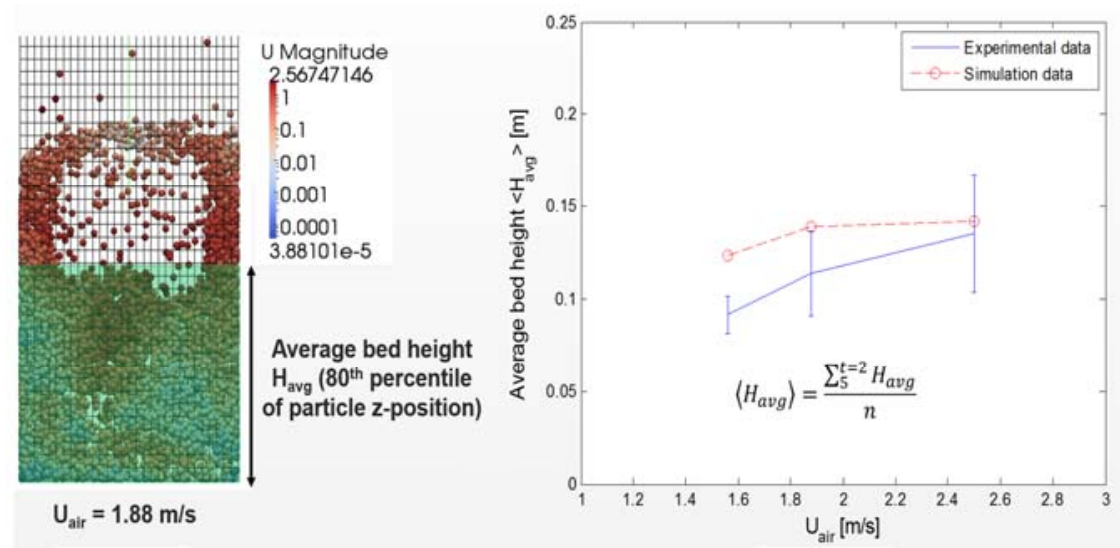

Figure 4: Estimation of $\mathrm{H}_{\text {avg }}$ for case with $\mathrm{U}_{\text {air }}$ of $1.88 \mathrm{~m} / \mathrm{s}$ (left) and comparison between experiments and simulation (right).

\subsection{Segregation patterns on a virtual destoner}

\subsubsection{Effect of fluidization}

The effect of fluidizing air velocity $\left(U_{f}\right)$ on the observed segregation patterns on a virtual destoner are shown in figure 5 (vectors of superficial air velocity magnitude). We have studied the system at constant vibrational conditions (amplitude of $1.5 \mathrm{~cm} / \mathrm{s})$ and deck inclination $(\theta=5$ degrees) with and without the effect of fluidization, i.e. at $U_{f}=0,1.5$ and $2.0 \mathrm{~m} / \mathrm{s}$ respectively (figure 5). 


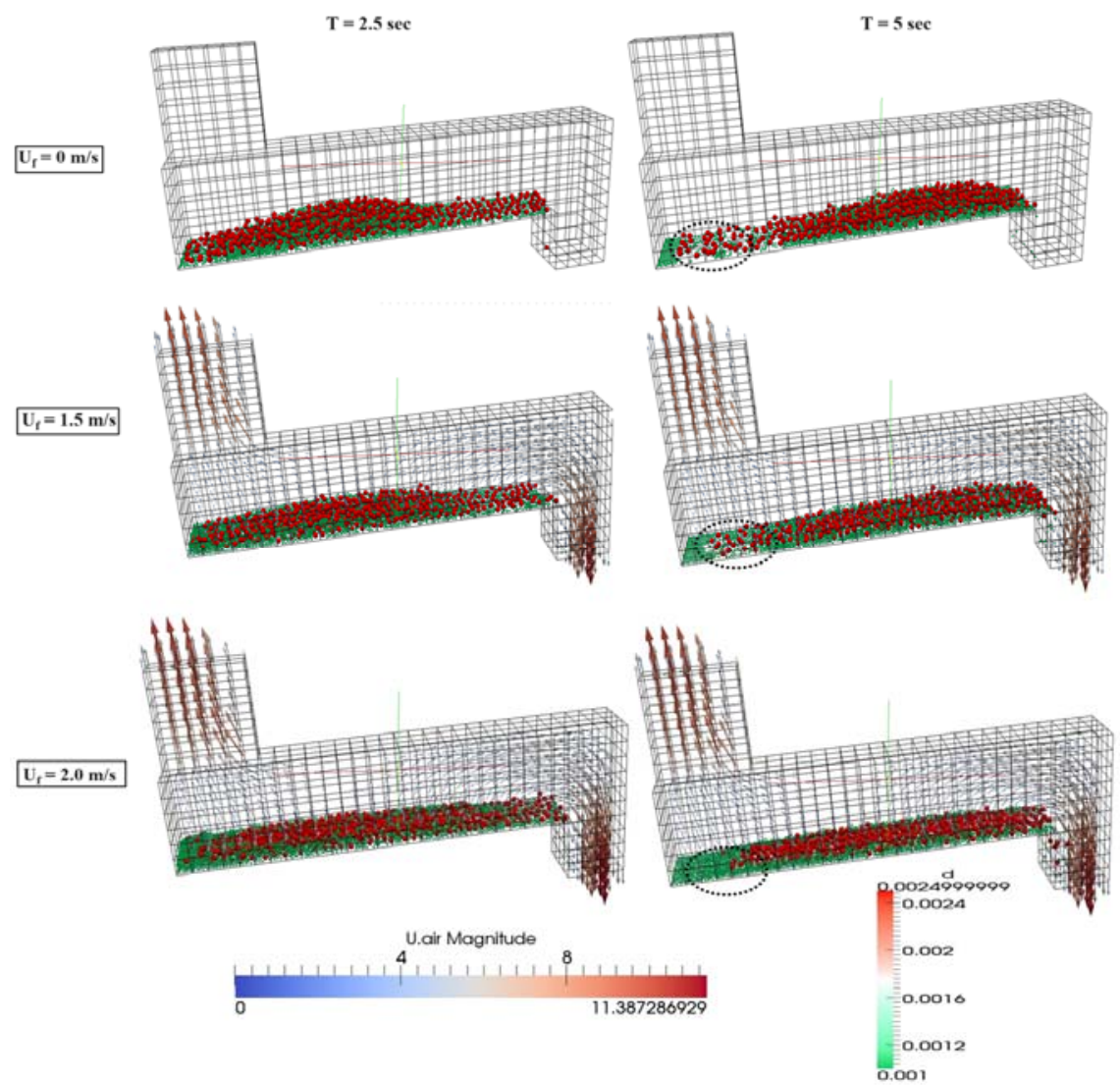

Figure 5: Effect of fluidization on segregation profiles at constant deck tilt and vibrational conditions (particles coloured by diameter): vectors of superficial air velocity magnitude at $\mathrm{t}=2.5$ and 5 seconds respectively.

When increasing the air velocity from 0 to $2 \mathrm{~m} / \mathrm{s}$; there is a notable increase in the separation between the lighter grains and stones (note the highlighted regions in figure 6). In the absence of fluidization all the material (stones and grains) are conveyed upwards (no density separation between the fractions). At superficial velocities closer to the minimum fluidization velocity of stones $\left(U_{m f}=1.4 \mathrm{~m} / \mathrm{s}\right)$, the lighter grains (with $U_{m f}$ in the range of 0.65 to $0.75 \mathrm{~m} / \mathrm{s}$ ) would be fluidized, while the heavier stones would be at the limit of fluidization and stay in contact with the vibrating deck and conveyed upwards. Hence, a sufficient fluidization velocity is needed in order to generate an optimal separation between the two fractions. The air velocities in the test cases were not increased beyond the $2.0 \mathrm{~m} / \mathrm{s}$ cut-off established by end users of real destoners, as at such higher velocities the air distribution system of the machine is at its limit (not an ideal scenario). 


\subsubsection{Effect of deck tilt}

The effect of deck inclinations $(\theta=5$ and 15 degrees respectively) on the segregation patterns on a virtual destoner at constant vibrational (amplitude of $1.5 \mathrm{~cm} / \mathrm{s})$ and fluidization conditions $\left(U_{f}=2.0 \mathrm{~m} / \mathrm{s}\right)$ are shown in figure 6 . The deck at 5 degrees is considered to be at optimal inclination (based on data from end-users of destoners). The slope provided in this deck is sufficient to enable the lighter grains (that are fluidized) to accumulate at the base, while the heavier stones can be propagated to the higher end and subsequently be removed from the system (i.e. the deck vibrational force component can overcome the corresponding gravitational component: $\left.F_{\text {vibration }}>F_{\text {gravity }}\right)$.

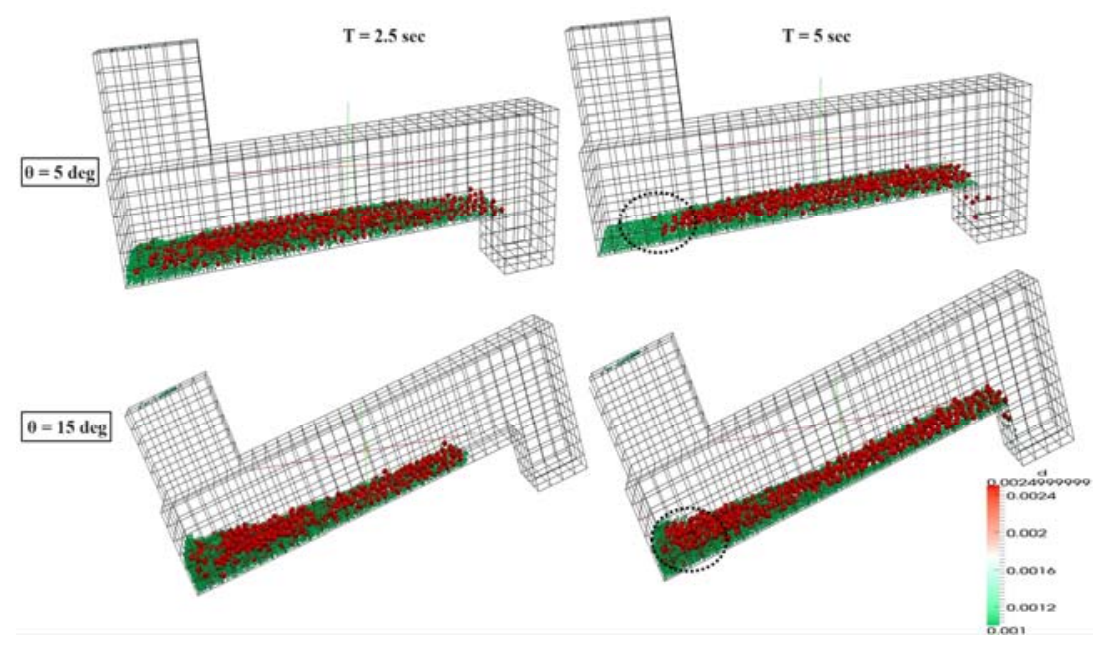

Figure 6: Effect of deck tilt on segregation profiles at constant vibrational and fluidization conditions (particles coloured by diameter): at $\mathrm{t}=2.5$ and 5 seconds respectively.

The development of a clear separation zone can be noticed at $\mathrm{t}=5$ seconds. When increasing the inclination to 15 degrees the observed separation between stones and food grains is poor as clearly indicated by the differences in the granular bed at $t=5$ seconds (note the highlighted regions in figure 5) This deviation from the optimal state can be attributed to the increased gravitational force component (due to increased slope of the deck) experienced by all the particles in the system leading to clumping of both stones and grains at the base of the deck (i.e. the deck vibrational force component cannot overcome the corresponding gravitational component : $\left.F_{\text {vibration }}<F_{\text {gravity }}\right)$.

\section{Conclusion}

A CFD-DEM framework has been proposed in this work to study the segregation of grains on a destoner. In this framework, the time discretized solutions for the instantaneous force balances around individual particles coupled 
with the Navier-Stokes equations are used to describe the overall contact dominated granular flow. The framework was validated using a fluidized-bed test case, with good agreement noted between simulations and experimental data. Furthermore, we utilized this method to demonstrate some of the general operating principles of a destoner, highlighting the importance of such frameworks in designing novel grain separation machinery. This fundamental study was able to demonstrate the development of a clear separation zone between stones and grains (modelled based on the micro-properties of rapeseed) on a virtual destoner. Fluidization and deck inclination were identified as the two critical parameters that affect the grain segregation, with fluidization conditions chosen close to the minimum fluidization velocity of stones. At these conditions, the stones are not fluidized and are carried to the higher side (steeper end) due to the vibrating action of the deck, while the fluidized grains move towards the lower end of the deck. In addition, the degree of inclination of the deck of a destoner is also critical to achieving acceptable segregation on it. A deck inclination of 5 degrees is considered optimal while steeper slopes (inclinations of 15 degrees) are deemed unsuitable for segregation as clumping of both stones and grains is noted at the base of the deck. Additionally, a fluidization velocity of $2.0 \mathrm{~m} / \mathrm{s}$ produced a good separation between the two fractions. These calculated process parameters are akin to the optimal operating parameters of a lab scale destoner $\left(U_{f}\right.$ of $2.25 \mathrm{~m} / \mathrm{s}$ and 5 degree tilt - data obtained from manufacturers) and further demonstrate the capabilities of a CFD-DEM framework in predicting segregation patterns on gravity separators.

Detailed validation (by comparing the numerical results with experimental data from destoners) of the implementation of this framework to describe gravity separators is warranted before acknowledging the results from these numerical simulations. Nevertheless the general behavioural trends are existent, and this model will form the basis for developing more quantitative approaches to allow further investigation of the separation mechanism and exploration of the effect of different physical and process parameters on the efficiency of grain separation in gravity separators.

\section{Acknowledgement}

This project is supported by the SEVENTH FRAMEWORK PROGRAMME of EU, Industry-Academia Partnerships and Pathways (IAPP) - Marie Curie Actions. Grant no.: 324433.

\section{References}

[1] Das, H., Separation of paddy and rice on an oscillating tray type separator, Journal of Agricultural Engineering Research, Vol. 34, pp. 85-95, 1986.

[2] Cundall, P.A. \& Strack, O.D.L., A discrete numerical model for granular assemblies, Géotechnique, pp. 47-65, 1979. 
[3] Zhu, H.P., Zhou, Z.Y., Yang, R.Y. \& Yu, A.B., Discrete particle simulation of particulate systems: Theoretical developments, Chemical Engineering Science, Vol. 62, pp. 3378-3396, 2007.

[4] Li, J., Webb, C., Pandiella, S.S. \& Campbell, G.M., A Numerical Simulation of Separation of Crop Seeds by Screening - Effect of Particle Bed Depth, Food and Bioproducts Processing, Vol. 80, pp. 109-117, 2002.

[5] Sakaguchi, E., Suzuki, M., Favier, J.F. \& Kawakami, S., PH - Postharvest Technology: Numerical Simulation of the Shaking Separation of Paddy and Brown Rice using the Discrete Element Method, Journal of Agricultural Engineering Research, Vol. 79, pp. 307-315, 2001.

[6] Tsuji, Y., Tanaka, T. \& Ishida, T., Lagrangian numerical simulation of plug flow of cohesionless particles in a horizontal pipe, Powder Technology, Vol. 71 , pp. 239-250, 1992.

[7] Crowe, C.T., Schwarzkopf, J.D., Sommerfeld, M. \& Tsuji, Y., Multiphase Flows with Droplets and Particles, Second Edition ed., CRC Press: Taylor \& Francis Group, pp. 120-132, 2012.

[8] Boac, J.M., Casada, M.E., Maghirang, R.G. \& Harner, J.P., Material and interaction properties of selected grains and oilseeds for modeling discrete particles, Transactions of the ASABE, Vol. 53, pp. 1201-1216, 2010.

[9] Goldschmidt, M.J.V., Link, J.M., Mellema, S. \& Kuipers, J.A.M., Digital image analysis measurements of bed expansion and segregation dynamics in dense gas-fluidised beds, Powder Technology, Vol. 138, pp. 135-159, 2003. 